\title{
Erratum: Coherent control of 40-THz optical phonons in diamond using femtosecond optical pulses [Phys. Rev. B 101, 174301 (2020)]
}

\author{
Tetsuya Kimata $\odot$, Kazuma Yoda, Hana Matsumoto, Hiroyuki Tanabe, Fujio Minami, \\ Yosuke Kayanuma, and Kazutaka G. Nakamura
}

(Received 10 November 2020; published 25 November 2020)

DOI: 10.1103/PhysRevB.102.179903

The purpose of this erratum is to correct a parameter error in our recently published paper [1]. The correct parameters that should be substituted into the following equation:

$$
E_{1}(t)=\sum_{k=1}^{5} E_{k} \exp \left(-\frac{t^{2}}{\sigma_{k}^{2}}\right) \cos \left[2 \pi\left(\Omega_{k}+\theta t\right) t\right]
$$

are $\left[E_{k}, \Omega_{k}(1 / \mathrm{fs}), \sigma_{k}(\mathrm{fs})\right]=(0.137,0.432,20.129),(0.183,0.405,30.480),(0.305,0.379,26.099),(0.238,0.354,26.664)$, and $(0.137,0.335,30.597)$, where $E_{k}, \Omega_{k}$, and $\sigma_{k}$ are the ratio of the electric-field strengths, the optical frequency, and the pulse width of each component.

We converted the intensity of the spectrum $I(\lambda)$ [Fig. 2(a) in the original paper] into the electric-field spectrum $E(\Omega)$ and then fitted $E(\Omega)$ using five Gaussian curves, where $\lambda$ is the wavelength and $\Omega$ is the optical frequency. The fitted parameters of the electric-field spectrum $E(\Omega)$ are then converted into the parameters of the electric field of the pulse $E(t)$. In the parameter conversion above, we used the equations that were obtained via Fourier transformation of the Gaussian pulse shape.

Figure 2(b) shows the electric-field intensity characteristic generated from the five Gaussian curves when the correct parameters were used; Fig. 2(b) is modified to match Fig. 2(b) in the original paper. The phonon amplitude that was simulated using these parameters was also recalculated, and the results are shown in Fig. 5; Fig. 5 is modified to match Fig. 5 in the original paper. These figures are almost identical to the corresponding figures in the published paper.

(b)

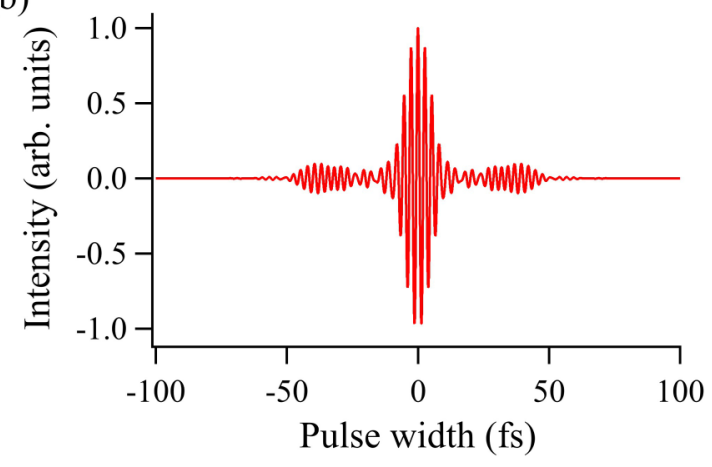

FIG. 2. (b) Electric-field intensity characteristic generated from the five Gaussian curves using the correct parameters. 


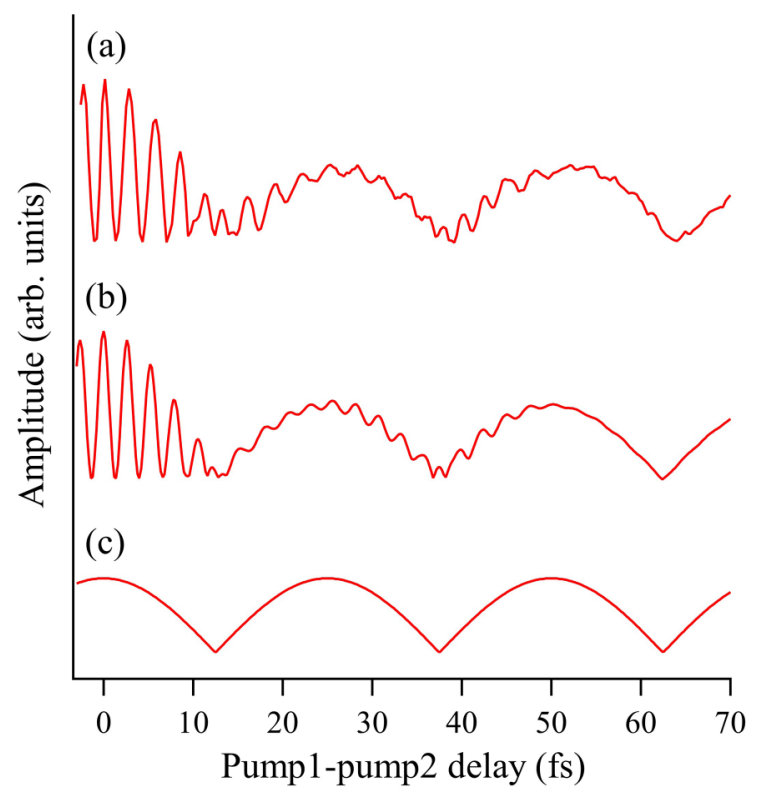

FIG. 5. (a) The measured amplitude of the controlled oscillation after pump 2 on the pulse overlap region. (b) The calculated phonon amplitude for all paths. (c) The calculated phonon amplitude for paths A and B. (This is the same caption that was given for the original Fig. 5.)

This erratum does not affect any of the conclusions presented in the published paper.

[1] T. Kimata, K. Yoda, H. Matsumoto, H. Tanabe, F. Minami, Y. Kayanuma, and K. G. Nakamura, Phys. Rev. B 101, 174301 (2020). 